\title{
Is percutaneous destruction of a solitary liver colorectal metastasis as effective as a resection?
}

\author{
Ugo Marchese ${ }^{1}$, Héloïse Seux ${ }^{1}$, Jonathan Garnier ${ }^{1}$, Jacques Ewald ${ }^{1}$, Gilles Piana ${ }^{2}$, Bernard Lelong ${ }^{1}$, \\ Cécile De Chaisemartin ${ }^{1}$, Hélène Meillat ${ }^{1}$, Jean-Robert Delpero ${ }^{1}$, and Olivier Turrini ${ }^{3}$ \\ ${ }^{1}$ Department of Surgery, Institut Paoli-Calmettes, ${ }^{2}$ Department of Radiology, Institut Paoli-Calmettes, \\ ${ }^{3}$ Department of Surgery, Institut Paoli-Calmettes, Aix-Marseille University, CNRS, Inserm, CRCM, Marseille, France
}

\begin{abstract}
Backgrounds/Aims: Surgical resection remains the gold standard in the treatment of colorectal liver metastasis. However, when a patient presents with a deep solitary colorectal liver metastasis (S-CLM), the balance between the hepatic volume sacrificed and the S-CLM volume is sometimes clearly unappropriated. Thus, alternatives to surgery, such as operative and percutaneous radiofrequency ablation (RFA) and microwave ablation (MWA), have been developed. This study aimed to identify the prognostic factors affecting survival of patients with S-CLM who undergo curative-intent liver resection or local destruction (RFA or MWA). Methods: We retrospectively identified 211 patients with synchronous or metachronous S-CLM who underwent either surgical resection $(n=182)$ or local destruction (RFA or MWA; $n=29$ ) according to the S-CLM size, location, and surrounding Glissonian structures. Results: Patients who underwent RFA or MWA had S-CLM of a smaller size than those who underwent resection (mean 19.7 vs. $37.3 \mathrm{~mm}, p<.01$ ). The 1-, 3-, and 5-year overall survival (OS) rates were $97.4 \%, 84.9 \%$, and $74.9 \%$, respectively. The 1-, 3-, and 5-year disease-free survival (DFS) rates were $77.9 \%, 47 \%$, and $38.9 \%$, respectively. S-CLM located in the left liver $(p=.04)$, S-CLM KRAS mutation $(p<.01)$, and extra-hepatic recurrence $(p<.01)$ were identified as independent poor risk factors for overall survival (OS); the OS and DFS were comparable in patients with surgical procedure or percutaneous MWA. Conclusions: In eligible S-CLM cases, percutaneous MWA seems to be as oncologically efficient as surgical resection and should be include in the decision-tree for treatment strategies. (Ann Hepatobiliary Pancreat Surg 2021;25:198-205)
\end{abstract}

Key Words: Hepatic resection; Liver metastases; Solitary; Microwave ablation; Percutaneous

\section{INTRODUCTION}

When feasible, surgical resection remains the gold standard in the treatment of colorectal liver metastasis (CLM). In a previous study, patients who underwent complete resection of initially unresectable bilobar CLM benefited from extreme strategies such as a two-stage hepatectomy. ${ }^{1}$ Moreover, the outcomes after parenchyma-sparing hepatectomy were comparable to those after major hepatectomy. ${ }^{2}$ Consequently, in the case of multiple peripheral CLM, parenchyma-sparing resection is preferred for reducing postoperative morbidity and sparing the parenchyma from the perspective of iterative hepatectomy. Inversely, among patients with hepatic disease spread, those with solitary CLM (S-CLM) that are present in a deep location may undergo major hepatectomy. In this situation, the balance between the hepatic volume sacrificed and the $\mathrm{S}-\mathrm{CLM}$ volume is sometimes clearly unappropriated. Alternatives to surgery, such as operative and percutaneous radiofrequency ablation (RFA) ${ }^{3}$ and microwave ablation (MWA), ${ }^{4}$ have been developed. While these techniques are useful for deep S-CLM in terms of hepatic parenchyma sparing, they have contrasting outcomes. These procedures are associated with increased local recurrence rates $^{5}$ and are not the first choice for curative-intent strategies; however, local destruction leads to reduced morbidity $^{6}$ and a reduced length of hospital stay ${ }^{7}$ compared with surgical resection. The choice between these treatment

Received: July 9, 2020; Revised: October 14, 2020; Accepted: October 15, 2020

Corresponding author: Olivier Turrini

Department of Surgery, Institut Paoli-Calmettes, Aix-Marseille University, CNRS, Inserm, CRCM, 232 boulevard Sainte Marguerite, Marseille 13009, France

Tel: +33-0491223660, Fax: +33-049223550, E-mail: turrinio@ipc.unicancer.fr

Copyright (C) 2021 by The Korean Association of Hepato-Biliary-Pancreatic Surgery

This is an Open Access article distributed under the terms of the Creative Commons Attribution Non-Commercial License (http://creativecommons.org/ licenses/by-nc/4.0) which permits unrestricted non-commercial use, distribution, and reproduction in any medium, provided the original work is properly cited. Annals of Hepato-Biliary-Pancreatic Surgery • pISSN: 2508-5778 - elSSN: 2508-5859 
types is crucial because many patients may benefit from adjuvant chemotherapy; ${ }^{8}$ major resection with poor postoperative outcomes may delay or prevent the administration of such treatment, directly affecting survival.

This study aimed to identify the prognostic factors affecting the recurrence in and survival of patients with S-CLM who undergo curative-intent liver resection or local destruction.

\section{MATERIALS AND METHODS}

\section{Patient selection}

From January 1, 2005 to June 31, 2018, 211 consecutive patients were retrospectively identified with a S-CLM and no extra-hepatic disease, and underwent a liver procedure at Institut Paoli-Calmettes (Marseille, France). All patient data were entered prospectively into a clinical database (NCT03686137) and the study was approved by the Institutional Review Board (PACA-0118). Patients eligible for the present study met the following criteria: a) had synchronous or metachronous S-CLM, and b) had curative-intent treatment (i.e., patients for whom local destruction was decided on instead of surgery owing to poor clinical status/severe comorbidities or reduced life expectancy were excluded). In addition, they had to provide written informed consent.

All patients were initially staged by a physical examination, thoraco-abdominal computerized tomography (CTscan), and carcinoembryonic antigen serum level. Owing to the period of inclusion, patients underwent routine liver magnetic resonance imaging but not positron emission tomography. After staging and before treatment initiation, all cases were discussed during a multidisciplinary board meeting, comprising an oncologist, at least one hepatic surgeon expert and one expert radiologist, for the appropriate selection of neoadjuvant chemotherapy or upfront resection/RFA/MWA. Criteria required for the achievement of local destruction were: non-sub-capsular location, S-CLM size $<30 \mathrm{~mm}$, absence of direct contact with a major Glissonian structure (i.e., left/right or sectorial Glissonian pedicles). If neoadjuvant chemotherapy was administered, patients were re-staged within 4 weeks before surgery. In patients with synchronous S-CLM, combined resection (i.e., colorectal resection and liver procedure) or staged resection was decided on.

\section{Surgical or percutaneous approach}

Owing to the period of inclusion, during the multidisciplinary board meeting, both hepatic surgeons and radiologists decided on the appropriate management of S-CLM using either a surgical (comprising resection (parenchymasparing hepatectomy [PSH] or non-PSH), or RFA) or percutaneous (MWA; introduced in our institution since 2012) approach. Liver resection could be achieved through laparotomy or a laparoscopic approach according to the surgeon's preference. A thorough abdominal exploration was first performed to eliminate carcinomatosis; intraoperative liver ultrasound was then routinely performed to confirm a) the uniqueness of the S-CLM and b) liver procedure to be used. Non-PSH procedures comprised hemi-hepatectomy or extended hemi-hepatectomy (after portal vein embolization); PSH included atypical resection, segmentectomy or bi-segmentectomy. Surgery was conducted without routine pedicular clampage, ${ }^{9}$ and all specimens were inked for the facilitation of margin assessment. Intraoperative RFA was performed with the Olympus Celon System ${ }^{\circledR}$ (Shinjuku-ku, Tokyo 163-0914, Japan). Abdominal drain was disposed only after extended hemi-hepatectomy. ${ }^{10}$

Percutaneous MWA under ultrasound or CT guidance was performed with the Ethicon Neuwave MWA System ${ }^{\circledR}$ (Ethicon US, LLS); patients were expected to be discharged home on the same day or the day after the procedure. Patients with percutaneous MWA could undergo resection of the colorectal cancer the day after MWA, consequently with a prolonged length of hospital stay.

\section{Study parameters}

The variables evaluated included age, sex, primary tumor location (i.e., colon or rectum), synchronous or metachronous disease, neoadjuvant treatment administration and number of cycles prior to S-CLM treatment, serum carcinoembryonic antigen level (UI/ml), segment and hemiliver (i.e., right or left) location of the S-CLM based on the tumor center, type of surgery (i.e., PSH or non-PSH), synchronous resection of primary tumor, morbidity (according to the Clavien-Dindo classification), ${ }^{11}$ 30-day mortality (or before hospital discharge), length of hospital stay, and readmission, as well as adjuvant treatment administration. Data on the margin status in patients who underwent surgical resection (a resection margin $<1 \mathrm{~mm}$ was considered 'involved' (R1)), ${ }^{12}$ S-CLM size (mm; based on 
specimen examination or preoperative imaging in patients who underwent RFA or MWA), and S-CLM mutational status (i.e., KRAS, NRAS, and BRAF mutations) were also recorded. Disease recurrence date and site (i.e., hepatic or extra-hepatic) were noted for disease-free survival (DFS) evaluation. The date of death (or the censor date: December 1,2018 ) was also recorded to permit the evaluation of overall survival (OS).

\section{Statistical analysis}

Data analyses were performed using GraphPad Prism software, version 5.0d (GraphPad Software Inc., La Jolla, CA) and SAS statistical software version 9.1 (SAS Institute, Inc., Cary, NC). Categorical factors were compared using Fisher's exact test, and continuous variables using Student's $t$ test. The association of categorical factors with survival was assessed using the Kaplan-Meier method and was tested using the Wilcoxon test. Statistical significance was set at $p<.05$. Prognostic factors with $p<.1$ in the univariable analysis or that are known to impact DFS or OS were entered into a multivariable regression model for the determination of independent predictors.

\section{RESULTS}

\section{Patient characteristics}

Among the 211 patients, 139 had a right-sided (65.9\%) and 72 a left-sided (34.1\%) S-CLM. Neoadjuvant chemotherapy was decided on and administered in 131 patients (62.1\%). A surgical approach was used in 195 patients (92.4\%), whereas a percutaneous approach with MWA was used in 16 patients $(7.6 \%)$. S-CLMs were located on average $28.3 \mathrm{~mm}$ (range 1-120) from the surface of the liver; MWA-treated S-CLMs were more deeply located (39.6 versus $26.4 \mathrm{~mm} ; p=.04)$. Of the patients with synchronous disease $(n=84), 52(61.9 \%)$ underwent simultaneous primary tumor resection (Table 1).

\section{Surgical or percutaneous approaches}

Among patients in whom a surgical approach was used ( $\mathrm{n}=195), \mathrm{PSH}$, non-PSH, and RFA were administered in $117(60 \%), 65(33.3 \%)$, and $13(6.7 \%)$ patients, respectively. The overall morbidity was $24.6 \%$ (grade $3-4$ morbidity $2.6 \%$ ) and there was an absence of mortality. The length of hospital stay was 9 days (range, 4-31 days), and
Table 1. Clinical characteristics of the study sample

\begin{tabular}{lc}
\hline Sex ratio M/F & 1.48 \\
Mean age $( \pm \mathrm{SD})$ (years) & $65.3( \pm 10.38)$ \\
Primary tumor & $143(67.8)$ \\
Colon $(\%)$ & $68(32.2)$ \\
Rectum $(\%)$ & $84(39.8)$ \\
Synchronous disease $(\%)$ & $48.6( \pm 103)$ \\
Mean serum CEA level (UI/ml) $( \pm \mathrm{SD})$ & \\
S-CLM location & $139(65.9)$ \\
Right liver $(\%)$ & 32 \\
Segment 5 & 28 \\
Segment 6 & 56 \\
Segment 7 & 23 \\
Segment 8 & $72(34.1)$ \\
Left liver $(\%)$ & 2 \\
Segment 1 & 28 \\
Segment 2 & 11 \\
Segment 3 & 31 \\
Segment 4 & $131(62.1)$ \\
Neoadjuvant chemotherapy $(\%)$ & $4(2-18)$ \\
Median number of cycles (range)
\end{tabular}

CEA, carcinoembryonic antigen; S-CLM, solitary colorectal liver metastasis; SD, standard deviation

the readmission rate was $1 \%$.

Among patients in whom a percutaneous approach was employed $(n=16)$, the overall morbidity was $6.2 \%$ (one patient experienced a grade 2 wound hematoma), and there was an absence of mortality. The mean length of hospital stay was 2 days (range, 1-5), and none of the patients experienced readmission.

In total, 142 patients $(67.3 \%)$ received adjuvant chemotherapy (Table 2).

\section{Pathologic findings}

The mean S-CLM size was $34.9 \mathrm{~mm}$ (range, 5-210 $\mathrm{mm}$ ); patients who underwent RFA or MWA had a smaller S-CLM size than those with resection (19.7 vs. 37.3 $\mathrm{mm}, p<.01)$. In patients with resection $(\mathrm{n}=182)$, $\mathrm{R} 1$ resection was used in 32 patients (17.6\%). Data on S-CLM mutation status were available in 108 patients $(51.2 \%)$; a KRAS mutation was identified in 42 patients (38.9\%), whereas NRAS and BRAF mutations were observed in three patients each.

\section{OS}

During the 50-month mean follow-up period, 42 patients $(19.9 \%)$ died from disease recurrence. The median OS time was not reached. The 1-, 3-, and 5-year OS rates 
Table 2. Surgical or percutaneous approaches, and postoperative courses

\begin{tabular}{|c|c|c|}
\hline & & $p$ value \\
\hline \multicolumn{3}{|l|}{ Surgical approach (\%) } \\
\hline Resection (\%) & $182(86.3)$ & \\
\hline Non-PSH & 65 & \\
\hline Right/Extended right hepatectomy & 53 & \\
\hline Left/Extended left hepatectomy & 12 & \\
\hline $\mathrm{PSH}$ & 117 & \\
\hline Bi-segmentectomy & 31 & \\
\hline Segmentectomy & 74 & \\
\hline Atypical resection & 12 & \\
\hline RFA $(\%)$ & $13(6.2)$ & \\
\hline Percutaneous approach (MWA) (\%) & $16(7.5)$ & \\
\hline Combined primary tumor resection (\%) & $52\left(61.9^{\mathrm{a}}\right)$ & \\
\hline Colon & 39 & \\
\hline Rectum & 13 & \\
\hline Morbidity (\%) & $49(23.2)$ & ns \\
\hline Resection & 48 & \\
\hline RFA & 0 & \\
\hline Percutaneous MWA (Table 1) & 1 & \\
\hline 30-day mortality (\%) & 0 & ns \\
\hline Length of hospital stay (days) $( \pm \mathrm{SD})$ & $10( \pm 5)$ & $<.01$ \\
\hline Resection & 11 & \\
\hline RFA & 2 & \\
\hline Percutaneous MWA & 8 & \\
\hline Readmission $(\%)$ & $2(0.9)$ & ns \\
\hline Resection & 2 & \\
\hline RFA & 0 & \\
\hline Percutaneous MWA & 0 & \\
\hline
\end{tabular}

${ }^{a}$ According to the number of synchronous diseases $\mathrm{SD}$, standard deviation; PSH, parenchyma-sparing hepatectomy; RFA, radiofrequency ablation; MWA, microwave ablation

were $97.4 \%, 84.9 \%$, and $74.9 \%$, respectively. No difference in OS was observed between the two approaches (i.e., surgical or percutaneous) in the entire population (Fig. 1; median survival time was not reached in the 2 groups) or only among patients with an S-CLM size $<3$ cm (Fig 2; median survival time was not reached in the 2 groups). In the multivariate analysis, S-CLM located in the left liver $(p=.04)$, S-CLM KRAS mutation presence $(p$ $<.01)$, and extra-hepatic recurrence $(p<.01)$ were found to poorly influence OS (Table 3).

\section{DFS}

Disease recurrence was identified in 110 patients (52.1\%), comprising 41 patients (37.3\%) with hepatic recurrence, 62 patients $(56.4 \%)$ with extra-hepatic recurrence (the lung was the most frequently observed site $(n=48))$, and seven with simultaneous hepatic and extra-hepatic recur-

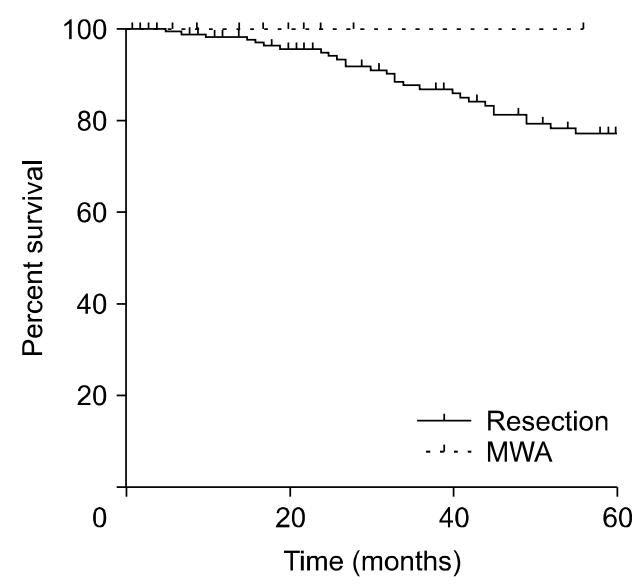

$\begin{array}{rcccccc}\text { Participants at risk } & & & & & & \\ \text { Time (months) } & 0 & 12 & 24 & 36 & 48 & 60 \\ \text { Resection } & 182 & 159 & 128 & 105 & 84 & 68 \\ \text { MWA } & 16 & 11 & 4 & 3 & 3 & 2\end{array}$

Fig. 1. Overall survival according to the adopted procedure (i.e. resection or percutaneous microwave ablation).

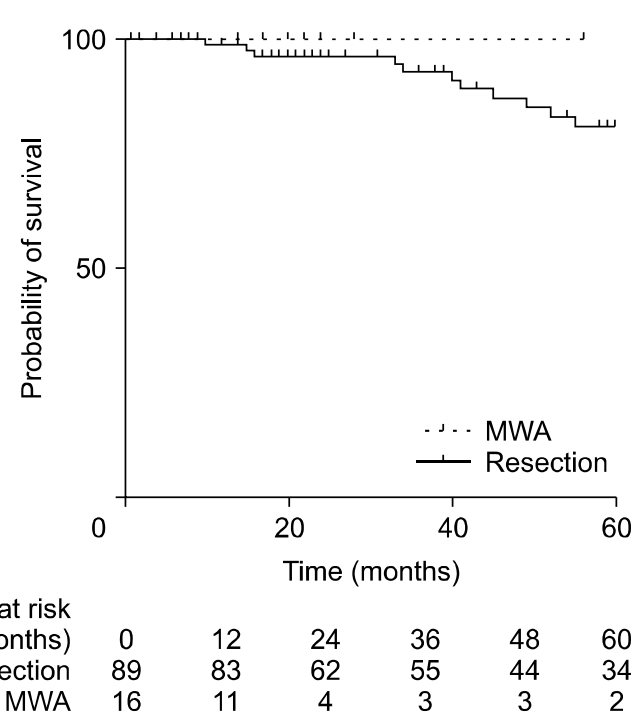

Fig. 2. Overall survival according to the adopted procedure (i.e. resection or percutaneous microwave ablation) in patients with $\mathrm{S}-\mathrm{CLM}<3 \mathrm{~cm}$.

rence. The median DFS time was 32 months. The 1-, 3-, and 5-year DFS rates were $77.9 \%, 47 \%$, and $38.9 \%$, respectively. In the univariate analysis, RFA $(p<.01)$ was the unique factor that poorly influenced DFS; none of the factors identified in the multivariate analysis independently influenced DFS.

\section{DISCUSSION}

In eligible patients with metachronous or synchronous S-CLM, we demonstrated an encouraging survival rate as- 
Table 3. Univariate and multivariate analyses of factors influencing overall survival

\begin{tabular}{lccc}
\hline & $p$ univariate & Hazard ratio & $p$ multivariate \\
\hline Synchronous disease & 0.28 & - & - \\
Primary tumor location & 0.42 & - & 0.04 \\
Serum CEA level & 0.12 & $1.92[1.01-3.65]$ & 0.25 \\
S-CLM location (right- versus left-sided) & 0.03 & $1.54[0.74-3.21]$ & $<0.01$ \\
Neoadjuvant chemotherapy & 0.07 & - & \\
Surgical approach & 0.38 & - & $<.97[1.54-10.2]$ \\
S-CLM size & 0.42 & - & - \\
R1 resection margin & 0.68 & -0.01 \\
KRAS mutation & 0.07 & 0.76 & \\
Adjuvant chemotherapy & 0.23 & $9.18[1.97-42.9]$ & $<0.01$ \\
Hepatic recurrence & $<0.01$ & & \\
Extra-hepatic recurrence & & & \\
\hline
\end{tabular}

CEA, carcinoembryonic antigen; S-CLM, solitary colorectal liver metastasis
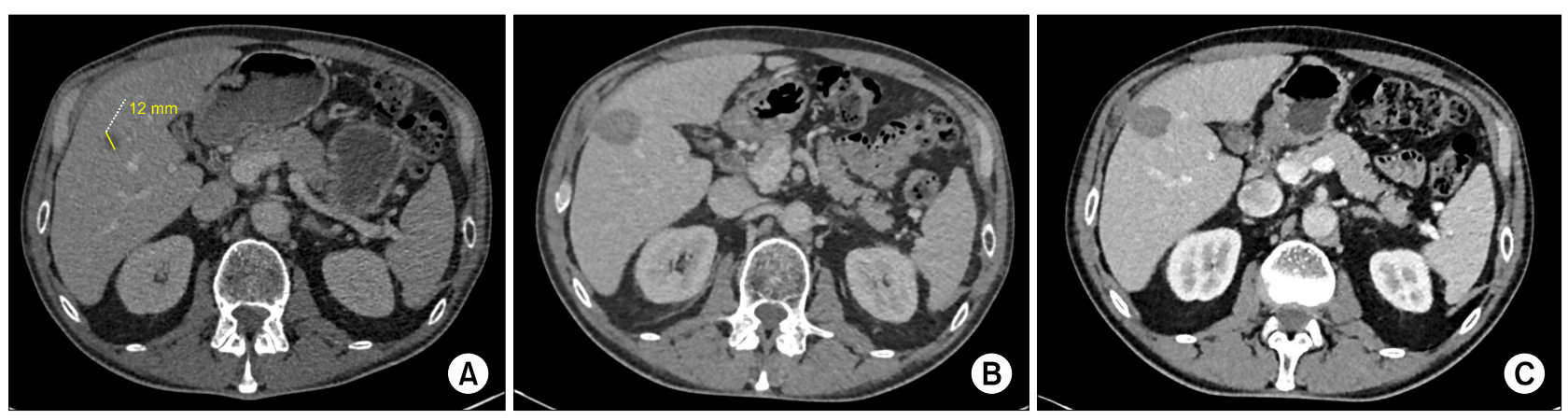

Fig. 3. (A) Patient diagnosed with a right colon cancer and a $12 \mathrm{~mm}$ synchronous S-CLM. A percutaneous microwave ablation (MWA) was achieved the day before a single port right hemicolectomy. The axial CT scan show the solitary metastasis prior to MWA. (B) The axial CT scan show the MWA result at 1 postoperative month. (C) The axial CT scan show the MWA result at 1 postoperative year.

sociated with local destruction.

\section{Strategy and survival}

In S-CLM treatment, surgery remains the gold standard, and, not surprisingly, we observed that resection was the preferred procedure $(86.3 \%)$ in our series. However, in the case of deep S-CLM, local destruction may be preferred for hepatic parenchyma sparing. The choice of local destruction can be debated from several aspects. In the case of disease spread, parenchyma-sparing procedures, whenever possible, should be preferred, as the high risk of hepatic recurrence (over $60 \%$ in the literature) ${ }^{13}$ may require iterative liver resection. In contrast, in S-CLM, the liver recurrence rate is relatively reduced ( $37 \%$ in our series), and a sparing procedure may not be the main concern for surgeons. However, parenchyma-sparing resection was preferred in our series $(60 \%)$ to eliminate useless paren- chyma sacrifice compared to the relatively small volume of the S-CLM (mean size $35 \mathrm{~mm}$ ). As all series on RFA report a worrying local recurrence rate, ${ }^{14}$ local destruction remains the preferred treatment only for fragile patients with S-CLM or in cases in which oncologic resection is impossible. However, available local destruction devices (changes in the RFA needles and generators used) or procedures (development of cryotherapy ${ }^{15}$ and MWA) have improved in the last two decades, and MWA is now an efficient oncologic procedure. ${ }^{16,17}$ Consequently, based on evidence that the efficiency of MWA could be oncologically comparable to that of resection in eligible patients (small $[<3 \mathrm{~cm}$ ] deep S-CLM without proximal Glissonian contact), we decided to perform percutaneous destruction. ${ }^{18}$ In the case of synchronous disease, this percutaneous approach was used preoperatively before primary colorectal resection (Fig. 3). With the use of this strategy, we ob- 
served neither morbidity nor mortality values that encourage the continuance of this low-impact strategy. In the same manner, the OS and DFS were not impacted by MWA, supporting the theory that this strategy is as oncologically efficient as surgical resection. Not surprisingly, we noted that intraoperative RFA poorly impacts DFS in the univariate analysis, as we theorized that intraoperative ultrasound guidance was probably less precise than percutaneous CT scan guidance, and we decided to abandon the use of such a procedure in the case of S-CLM.

In 1984, Wagner et al. ${ }^{19}$ showed that untreated S-CLM was associated with a median survival time of 2 years. In our series, after more than 4 years of follow-up, we observed an unreached median survival time and a 5-year OS rate of $75 \%$, underlining the major progress in the fields of CLM radiological evaluation, hepatic surgery and perioperative treatment in the last three decades. We observed comparable survivals rate irrespective of the approach (i.e., resection or local destruction), reinforcing our preference for percutaneous local destruction under CT scan guidance in patients with S-CLM.

It has been shown that synchronous disease ${ }^{20}$ and rightsided primary tumors ${ }^{21}$, which are supposedly associated with a greater level of KRAS mutations, are poor prognostic factors. However, synchronous disease or primary tumor location were not independently identified as factors influencing OS and/or DFS in our series. Similarly, perioperative treatment was not identified as an independent factor impacting OS or DFS. Because there is strong evidence for the use of perioperative chemotherapy for multiple $\mathrm{CLM}^{22}$ the use of this modality did not seem to be influential in patients with S-CLM (even those with synchronous disease, as previously reported by other series). ${ }^{23}$

\section{Left-sided S-CLM and KRAS mutation}

Several series have shown a higher number of right-sided CLM than left-sided CLM. ${ }^{24}$ Investigations on the asymmetrical distribution of CLM have not been able to identify a relationship between primary colorectal cancer and liver metastasis location. However, this could be explained by a) the higher right liver volume (approximately two-third of the total liver volume), and b) the constant anatomical angle of the left portal vein branch compared to the alignment of the right portal vein branch, which favors the preferential draining of the metastatic cancer cells to the right hemi-liver. According to these two points, we observed a larger number of right-sided S-CLM $(66 \%$ versus $34 \%)$. However, we noted that left-sided S-CLM was independently associated with poorer prognosis. Available data on the prognostic impact of the CLM hemi-liver location is insufficient, despite many small series reporting poorer prognoses. ${ }^{25-27}$ The mechanisms of metastatic cancer cell dissemination and implantation are unclear, and no indisputable conclusions can be drawn based on the current knowledge.

In this study, the occurrence of KRAS mutation was not rare $(39 \%)$ in S-CLM cases with resection, and the corresponding effect on survival and extra-hepatic recurrence was poor. The negative impact of KRAS mutations in CLM has already been reported; ${ }^{28}$ it could be a useful tool to be considered in patient treatment strategies. Indeed, patients should be treated with a liver procedure with the lowest possible impact (i.e., surgery or local destruction) for a favorable postoperative treatment outcome. For example, an eligible patient with S-CLM in a deep location may benefit from percutaneous local destruction to ensure low postoperative morbidity and high likelihood of prompt recovery so as to allow for the administration of adjuvant chemotherapy, if recommended according to the presence of poor prognostic factors (such as the presence of a concomitant BRAF mutation). ${ }^{29}$

\section{Limitations}

Our study has some limitations that must be highlighted. First, the retrospective design of the study has its own corresponding drawbacks. Second, changes in the perioperative treatments, devices used for local destruction (MWA was introduced since 2012 in our institution whereas RFA was historically used), as well as the global strategies through the relatively large period of inclusion may have impacted our results. Third, this study was not protocol-driven, so it introduced unforeseen factors. Nonetheless, the sample size is not considered small in this specific setting of S-CLM, which allows for the overriding of these limitations.

Patients with S-CLM had good prognoses, with a 5 -year OS rate of $75 \%$. In such settings, the efficacy of MWA seems to be oncologically comparable to that of resection. Thus, percutaneous MWA, when feasible, may 
be the procedure of choice to allow for prompt patient recovery and the administration of adjuvant chemotherapy.

\section{ACKNOWLEDGEMENTS}

We would like to thank Editage (www.editage.com) for English language editing.

\section{CONFLICT OF INTEREST}

The authors declare that they have no conflict of interest.

This research did not receive any specific grant from funding agencies in the public, commercial, or not-for profit sectors.

\section{ORCID}

Ugo Marchese: https://orcid.org/0000-0002-8647-7584

Héloïse Seux: https://orcid.org/0000-0002-8813-4032

Jonathan Garnier: https://orcid.org/0000-0001-5481-2926

Jacques Ewald: https://orcid.org/0000-0003-0286-0437

Gilles Piana: https://orcid.org/0000-0003-2220-8294

Bernard Lelong: https://orcid.org/0000-0003-1642-2913

Cécile De Chaisemartin: https://orcid.org/0000-0001-6834-8378

Hélène Meillat: https://orcid.org/0000-0003-4548-6481

Jean-Robert Delpero: https://orcid.org/0000-0002-0000-1332

Olivier Turrini: https://orcid.org/0000-0002-2144-2380

\section{AUTHOR CONTRIBUTIONS}

Conceptualization: UM, HS, JE, OT. Data curation: UM, HS, JG, OT. Formal analysis: UM, HS, JG, GP, OT. Writing - original draft: UM, HS, JG, JE, GP. Writing review \& editing: GP, BL, CDC, HM, JRD, OT.

\section{REFERENCES}

1. Moris D, Ronnekleiv-Kelly S, Kostakis ID, Tsilimigras DI, Beal EW, Papalampros A, et al. Operative results and oncologic outcomes of associating liver partition and portal vein ligation for staged hepatectomy (ALPPS) versus two-stage hepatectomy (TSH) in patients with unresectable colorectal liver metastases: a systematic review and meta-analysis. World J Surg 2018;42: 806-815.

2. Donadon M, Cescon M, Cucchetti A, Cimino M, Costa G, Pesi $\mathrm{B}$, et al. Parenchymal-sparing surgery for the surgical treatment of multiple colorectal liver metastases is a safer approach than major hepatectomy not impairing patients' prognosis: a bi-institutional propensity score-matched analysis. Dig Surg 2018;35:342349.

3. McGahan JP, Browning PD, Brock JM, Tesluk H. Hepatic ablation using radiofrequency electrocautery. Invest Radiol 1990;25: 267-270.

4. Shibata T, Niinobu T, Ogata N. Comparison of the effects of in-vivo thermal ablation of pig liver by microwave and radiofrequency coagulation. J Hepatobiliary Pancreat Surg 2000;7:592598.

5. White RR, Avital I, Sofocleous CT, Brown KT, Brody LA, Covey A, et al. Rates and patterns of recurrence for percutaneous radiofrequency ablation and open wedge resection for solitary colorectal liver metastasis. J Gastrointest Surg 2007;11:256-263.

6. Lee WS, Yun SH, Chun HK, Lee WY, Kim SJ, Choi SH, et al. Clinical outcomes of hepatic resection and radiofrequency ablation in patients with solitary colorectal liver metastasis. J Clin Gastroenterol 2008;42:945-949.

7. Oshowo A, Gillams A, Harrison E, Lees WR, Taylor I. Comparison of resection and radiofrequency ablation for treatment of solitary colorectal liver metastases. Br J Surg 2003;90: 1240-1243.

8. Nordlinger B, Sorbye H, Glimelius B, Poston GJ, Schlag PM, Rougier $\mathrm{P}$, et al. Perioperative FOLFOX4 chemotherapy and surgery versus surgery alone for resectable liver metastases from colorectal cancer (EORTC 40983): long-term results of a randomised, controlled, phase 3 trial. Lancet Oncol 2013;14:12081215.

9. Belghiti J, Noun R, Zante E, Ballet T, Sauvanet A. Portal triad clamping or hepatic vascular exclusion for major liver resection. A controlled study. Ann Surg 1996;224:155-161.

10. Brauer DG, Nywening TM, Jaques DP, Doyle MB, Chapman $\mathrm{WC}$, Fields RC, et al. Operative site drainage after hepatectomy: a propensity score matched analysis using the American College of Surgeons NSQIP targeted hepatectomy database. J Am Coll Surg 2016;223:774-783.e2.

11. Dindo D, Demartines N, Clavien PA. Classification of surgical complications: a new proposal with evaluation in a cohort of 6336 patients and results of a survey. Ann Surg 2004;240:205213.

12. Rajaganeshan R, Prasad R, Guillou PJ, Chalmers CR, Scott N, Sarkar R, et al. The influence of invasive growth pattern and microvessel density on prognosis in colorectal cancer and colorectal liver metastases. Br J Cancer 2007;96:1112-1117.

13. de Jong MC, Pulitano C, Ribero D, Strub J, Mentha G, Schulick $\mathrm{RD}$, et al. Rates and patterns of recurrence following curative intent surgery for colorectal liver metastasis: an international multi-institutional analysis of 1669 patients. Ann Surg 2009;250: 440-448.

14. Bai H, Huangz X, Jing L, Zeng Q, Han L. The effect of radiofrequency ablation vs. liver resection on survival outcome of colorectal liver metastases (CRLM): a meta-analysis. Hepatogastroenterology 2015;62:373-377.

15. Ng KM, Chua TC, Saxena A, Zhao J, Chu F, Morris DL. Two decades of experience with hepatic cryotherapy for advanced colorectal metastases. Ann Surg Oncol 2012;19:1276-1283.

16. Takahashi H, Kahramangil B, Kose E, Berber E. A comparison of microwave thermosphere versus radiofrequency thermal ablation in the treatment of colorectal liver metastases. HPB (Oxford) 2018;20:1157-1162.

17. Shady W, Petre EN, Do KG, Gonen M, Yarmohammadi H, Brown KT, et al. Percutaneous microwave versus radiofrequency ablation of colorectal liver metastases: ablation with clear margins (A0) provides the best local tumor control. J Vasc Interv 
Radiol 2018;29:268-275.e1.

18. Puijk RS, Ruarus AH, Vroomen LGPH, van Tilborg AAJM, Scheffer HJ, Nielsen K, et al. Colorectal liver metastases: surgery versus thermal ablation (COLLISION)- a phase III single-blind prospective randomized controlled trial. BMC Cancer 2018; $18: 821$.

19. Wagner JS, Adson MA, Van Heerden JA, Adson MH, Ilstrup DM. The natural history of hepatic metastases from colorectal cancer. A comparison with resective treatment. Ann Surg 1984; 199:502-508.

20. Goffredo P, Utria AF, Beck AC, Chun YS, Howe JR, Weigel $\mathrm{RJ}$, et al. The prognostic impact of KRAS mutation in patients having curative resection of synchronous colorectal liver metastases. J Gastrointest Surg 2019;23:1957-1963.

21. Creasy JM, Sadot E, Koerkamp BG, Chou JF, Gonen M, Kemeny NE, et al. The impact of primary tumor location on long-term survival in patients undergoing hepatic resection for metastatic colon cancer. Ann Surg Oncol 2018;25:431-438.

22. Van Cutsem E, Cervantes A, Adam R, Sobrero A, Van Krieken $\mathrm{JH}$, Aderka D, et al. ESMO consensus guidelines for the management of patients with metastatic colorectal cancer. Ann Oncol 2016;27:1386-1422.

23. Acciuffi S, Meyer F, Bauschke A, Settmacher U, Lippert H, Croner R, et al. Analysis of prognostic factors after resection of solitary liver metastasis in colorectal cancer: a 22 -year bicentre study. J Cancer Res Clin Oncol 2018;144:593-599.

24. Hosokawa I, Allard MA, Mirza DF, Kaiser G, Barroso E, Lapointe $\mathrm{R}$ et al. Outcomes of parenchyma-preserving hepatectomy and right hepatectomy for solitary small colorectal liver metastasis: a LiverMetSurvey study. Surgery 2017;162:223-232.

25. Shin H, Kim CW, Lee JL, Yoon YS, Park IJ, Lim SB, et al. Solitary colorectal liver metastasis after curative intent surgery: prognostic factors affecting outcomes and survival. ANZ J Surg 2019;89:61-67.

26. Ou S, Xu R, Li K, Chen $\mathrm{Y}$, Kong $\mathrm{Y}$, Liu $\mathrm{H}$, et al. Radiofrequency ablation with systemic chemotherapy in the treatment of colorectal cancer liver metastasis: a 10-year single-center study. Cancer Manag Res 2018;10:5227-5237.

27. Pathak S, Palkhi E, Dave R, White A, Pandanaboyana S, Prasad $\mathrm{KR}$, et al. Relationship between primary colorectal tumour and location of colorectal liver metastases. ANZ J Surg 2016;86:408410.

28. Shady W, Petre EN, Vakiani E, Ziv E, Gonen M, Brown KT, et al. Kras mutation is a marker of worse oncologic outcomes after percutaneous radiofrequency ablation of colorectal liver metastases. Oncotarget 2017;8:66117-66127.

29. Deshwar A, Margonis GA, Andreatos N, Barbon C, Wang J, Buettner $\mathrm{S}$, et al. Double $K R A S$ and $B R A F$ mutations in surgically treated colorectal cancer liver metastases: an international, multi-institutional case series. Anticancer Res 2018;38:2891-2895. 